\title{
IMPLICIT DIFFERENCE METHODS FOR NONLINEAR FIRST ORDER PARTIAL FUNCTIONAL DIFFERENTIAL SYSTEMS
}

Abstract. Initial problems for nonlinear hyperbolic functional differential systems are considered. Classical solutions are approximated by solutions of suitable quasilinear systems of difference functional equations. The numerical methods used are difference schemes which are implicit with respect to the time variable. Theorems on convergence of difference schemes and error estimates of approximate solutions are presented. The proof of the stability is based on a comparison technique with nonlinear estimates of the Perron type. Numerical examples are given.

1. Introduction. For any metric spaces $X$ and $Y$ we denote by $C(X, Y)$ the class of all continuous functions from $X$ into $Y$. If $A \subset X$ and $\alpha \in$ $C(X, Y)$ then $\left.\alpha\right|_{A}$ denotes the restriction of $\alpha$ to the set $A$. We will use vectorial inequalities with the understanding that the same inequalities hold between their corresponding components.

Suppose that $M=\left(M_{1}, \ldots, M_{n}\right) \in \mathbb{R}_{+}^{n}, a>0, \mathbb{R}_{+}=[0,+\infty), b=$ $\left(b_{1}, \ldots, b_{n}\right), b \in \mathbb{R}_{+}^{n}$ and $b>M a$. Let $E$ be the Haar pyramid

$$
E=\left\{(t, x) \in \mathbb{R}^{1+n}: t \in[0, a],-b+M t \leq x \leq b-M t\right\}
$$

where $x=\left(x_{1}, \ldots, x_{n}\right)$. Write $E_{0}=\left[-b_{0}, 0\right] \times[-b, b]$ where $b_{0} \in \mathbb{R}_{+}$and

$$
\begin{aligned}
B & =\left[-b_{0}-a, 0\right] \times[-2 b, 2 b], \\
E_{t} & =\left(E_{0} \cup E\right) \cap\left(\left[-b_{0}, t\right] \times \mathbb{R}^{n}\right), \quad 0 \leq t \leq a .
\end{aligned}
$$

For $(t, x) \in E$ we define

$$
D[t, x]=\left\{(\tau, y) \in \mathbb{R}^{1+n}: \tau \leq 0 \text { and }(t+\tau, x+y) \in E_{0} \cup E\right\} .
$$

2010 Mathematics Subject Classification: 35F25, 41A65, 65Q10.

Key words and phrases: functional differential equations, Haar pyramid, implicit difference methods. 
Given a function $z: E_{0} \cup E \rightarrow \mathbb{R}^{k}$ and a point $(t, x) \in E$, we consider the function $z_{(t, x)}: D[t, x] \rightarrow \mathbb{R}^{k}$ defined by $z_{(t, x)}(\tau, y)=z(t+\tau, x+y),(\tau, y) \in$ $D[t, x]$. Thus $z_{(t, x)}$ is the restriction of $z$ to the set $\left(E_{0} \cup E\right) \cap\left(\left[-b_{0}, t\right] \times \mathbb{R}^{n}\right)$, shifted to the set $D[t, x]$.

Put $\Omega=E \times C\left(B, \mathbb{R}^{k}\right) \times \mathbb{R}^{n}$ and suppose that $f: \Omega \rightarrow \mathbb{R}^{k}, f=$ $\left(f_{1}, \ldots, f_{k}\right)$, is a given function of the variables $(t, x, w, q), w=\left(w_{1}, \ldots, w_{k}\right)$, $q=\left(q_{1}, \ldots, q_{n}\right)$. We will say that $f$ satisfies the condition $(V)$ if for $(t, x, q) \in$ $E \times \mathbb{R}^{n}$ and $w, \bar{w} \in C\left(B, \mathbb{R}^{k}\right)$ such that $\left.w\right|_{D[t, x]}=\left.\bar{w}\right|_{D[t, x]}$ we have $f(t, x, w, q)$ $=f(t, x, \bar{w}, q)$. The condition $(V)$ means that the value of $f$ at $(t, x, w, q) \in \Omega$ depends on $(t, x, q)$ and on the restriction of $w$ to $D[t, x]$ only.

Let us denote by $z=\left(z_{1}, \ldots, z_{k}\right)$ an unknown function of the variables $(t, x)$. Given $\varphi: E_{0} \rightarrow \mathbb{R}^{k}$, we consider the system of functional differential equations

$$
\partial_{t} z_{i}(t, x)=f_{i}\left(t, x, z_{(t, x)}, \partial_{x} z_{i}(t, x)\right), \quad i=1, \ldots, k,
$$

with the initial condition

$$
z(t, x)=\varphi(t, x) \quad \text { on } E_{0}
$$

where $\partial_{x} z_{i}=\left(\partial_{x_{1}} z_{i}, \ldots, \partial_{x_{n}} z_{i}\right)$. System (1.1) has the property that every equation contains the vector of unknown functions and the derivatives of only one scalar function. We consider classical solutions of (1.1), 1.2 and we assume that $f$ satisfies the condition $(V)$. The Haar pyramid is a natural domain for the existence and uniqueness of classical or generalized solutions for nonlinear hyperbolic systems with initial conditions ([3], [12], [21]).

The following methods of construction of approximate solutions for nonlinear hyperbolic functional differential equations are known: the numerical method of bicharacteristics, the Euler difference method, the Lax scheme. The aim of this paper is to add a new element to the above sequence of numerical methods.

We are interested in establishing a method of numerical approximation of solutions to (1.1), (1.2) by means of solutions of associated systems of implicit difference functional equations and in estimating the difference between the exact and approximate solutions.

We first give some motivations for our investigations. Let $\left(h_{0}, h^{\prime}\right)=h$, $h^{\prime}=\left(h_{1}, \ldots, h_{n}\right)$, stand for steps of a mesh. Let $\left(t^{(r)}, x^{(m)}\right), m=\left(m_{1}, \ldots, m_{n}\right)$, denote nodal points. Let $E_{0 . h}$ and $E_{h}$ be the sets of all nodal points which are elements of $E_{0}$ and $E$ respectively. Solutions of difference equations are defined on $E_{0 . h} \cup E_{h}$. Classical difference methods for (1.1), (1.2) consist in replacing the partial derivatives $\partial_{t}$ and $\left(\partial_{x_{1}}, \ldots, \partial_{x_{n}}\right)=\partial_{x}$ with difference operators $\delta_{0}$ and $\left(\delta_{1}, \ldots, \delta_{n}\right)=\delta$. Moreover, system (1.1) contains the functional variable $z_{(t, x)}$ which is an element of $C\left(D[t, x], \mathbb{R}^{k}\right)$. Then we need an 
interpolating operator

$$
T_{h}: \mathcal{F}\left(E_{0 . h} \cup E_{h}, \mathbb{R}^{k}\right) \rightarrow C\left(E_{0} \cup E, \mathbb{R}^{k}\right) .
$$

Additional assumptions on $T_{h}$ will be needed in Section 4. System (1.1) leads to the difference functional system

$$
\delta_{0} z_{i}^{(r, m)}=f_{i}\left(t^{(r)}, x^{(m)},\left(T_{h} z\right)_{\left(t^{(r)}, x^{(m)}\right)}, \delta z_{i}^{(r, m)}\right), \quad i=1, \ldots, k,
$$

where $\delta z_{i}=\left(\delta_{1} z_{i}, \ldots, \delta_{n} z_{i}\right)$. Initial conditions are associated with (1.4).

The following examples of systems (1.4) are considered in the literature: the Euler difference method and the Lax scheme (see [12, Chapter 3]). Two types of assumptions are needed in theorems on the stability of difference schemes generated by (1.1), 1.2. Conditions the first type concern the regularity of $f$ and they are the same for both methods. It is required that the function $f$ of variables $(t, x, w, q)$ satisfies nonlinear estimates of the Perron type with respect to $w$ and it is of class $C^{1}$ with respect to $q$ and that the functions $\partial_{q} f_{i}=\left(\partial_{q_{1}} f_{i}, \ldots, \partial_{q_{n}} f_{i}\right), 1 \leq i \leq k$, are bounded on $\Omega$. Assumptions the second type in convergence theorems are the Courant-Friedrichs-Levy (CFL) conditions (see [8, Chapter III], [9], [12, Chapter III]). For the analysis of the stability of the Euler difference method we need the following (CFL) conditions:

(i) for each $P=(t, x, w, q) \in \Omega$ we have

$$
1-h_{0} \sum_{j=1}^{n} \frac{1}{h_{j}}\left|\partial_{q_{j}} f_{i}(P)\right| \geq 0, \quad 1 \leq i \leq k,
$$

(ii) for each $1 \leq i \leq k$, the function

$$
\operatorname{sign} \partial_{q} f_{i}=\left(\operatorname{sign} \partial_{q_{1}} f_{i}, \ldots, \operatorname{sign} \partial_{q_{n}} f_{i}\right)
$$

is constant on $\Omega$.

The (CFL) conditions for (1.1) and for the Lax difference scheme have the form

$$
\frac{1}{n}-\frac{h_{0}}{h_{j}}\left|\partial_{q_{j}} f_{i}(P)\right| \geq 0, \quad 1 \leq j \leq n, 1 \leq i \leq k,
$$

where $P \in \Omega$.

Note that the assumptions (1.5) and (1.7) require some relations between $h_{0}$ and $h^{\prime}$. We conclude from condition (ii) that we need more restrictive assumptions on $f$ for the Euler method than for the Lax scheme.

Of course there are systems (1.1) for which both the difference methods are convergent. It follows from the theory of bicharacteristics that in this case the Euler method is more suitable than the Lax scheme. This theoretical observation is confirmed by numerical experiments. 
With the above motivation we are interested in proving convergence results for Euler methods and for a possibly large class of nonlinear problems. More precisely, we will show that there are convergent difference methods of the Euler type for which the assumption that the functions (1.6) are constant can be omitted. In other words, we show that we do not need the Lax difference schemes for systems (1.1) with natural regularity assumptions on $f$. Since we consider implicit difference schemes, we show that the (CFL) condition 1.5 can also be omitted in the convergence analysis.

In recent years, a number of papers concerning numerical methods for first order partial functional differential equations have been published. Explicit difference schemes for initial or initial boundary value problems were studied in the papers [11, [20] and in the monograph [12. The proofs of convergence were based on functional difference inequalities or on general theorems on error estimates for approximate solutions to functional difference equations of the Volterra type with an unknown function of several variables.

Sufficient conditions for the convergence of implicit difference schemes for initial boundary value problems are given in [4], 13]. It is assumed in [4] that the functions (1.6) are constant on $\Omega$. It follows that the results of [4] are not applicable to (1.1), 1.2). The papers [16], [17] initiated investigations of implicit difference schemes for parabolic equations. Monotone iterative methods and implicit difference schemes for computing approximate solutions of parabolic equations with time delay were analysed in [18], [19], 27]. Implicit difference schemes for nonlinear parabolic functional differential equations with initial boundary conditions of the Dirichlet type were studied in [14]. Convergence theorems were proved by using a comparison technique.

In this paper we propose a new class of difference schemes corresponding to (1.1), (1.2). We approximate the unknown function $z$ and its partial derivatives $\partial_{x} z_{i}, 1 \leq i \leq k$, by solutions of quasilinear systems of difference functional equations which are implicit with respect to the variable $t$. In this procedure we linearize (1.1) with respect to the last variable. This method has been used in the existence and uniqueness theory for classical or generalized solutions.

Sufficient conditions for the existence and uniqueness of classical or generalized solutions can be found in the papers [2], 3], [10, [21], 22] and the monograph [12]. We use general ideas concerning the stability of numerical methods for evolution differential or functional differential equations, introduced in [15], [23], 24].

First order partial functional differential equations find applications in different fields of knowledge. Differential-integral systems have been proposed in [1] as simple mathematical models for the nonlinear phenomenon 
of harmonic generation of laser radiation through piezoelectric crystals for nondispersive materials and of Maxwell-Hopkinson type. Systems of differential equations containing operators acting on the unknown density of populations in dependence on their age, size, and DNA content, are considered in [25. An equation with a deviated argument ([6]) describes the density of households at a time $t$, depending on their estate, in the theory of the distribution of wealth. Another system of integral-differential equations is used in mathematical biology to investigate an age-dependent epidemic of a disease with vertical transmissions [7]. The paper [26] deals with integral differential equations motivated by applications in the theory of screening of granular bodies. For further information on applications of functional differential equations see the monographs [12], [28].

2. Discretization of nonlinear systems. We will denote by $\mathcal{F}(X, Y)$ the class of all functions from $X$ into $Y$ where $X$ and $Y$ are arbitrary sets. We will use the symbols $\mathbb{N}$ and $\mathbb{Z}$ to denote the sets of natural numbers and integers respectively. Denote by $M_{k \times n}$ the class of all $k \times n$ matrices with real entries. For $x \in \mathbb{R}^{n}, p \in \mathbb{R}^{k}, U \in M_{k \times n}$ where $x=\left(x_{1}, \ldots, x_{n}\right)$, $p=\left(p_{1}, \ldots, p_{k}\right), U=\left[u_{i j}\right]_{i=1, \ldots, k, j=1, \ldots, n}$ we put $\|x\|=\left|x_{1}\right|+\cdots+\left|x_{n}\right|$, $\|p\|_{\infty}=\max \left\{\left|p_{i}\right|: 1 \leq i \leq k\right\}$ and

$$
\|U\|=\max \left\{\sum_{j=1}^{n}\left|u_{i j}\right|: 1 \leq i \leq k\right\} .
$$

The product of two matrices is denoted by $\star$. If $U \in M_{k \times n}$ then $U^{T}$ denotes the transpose matrix. The scalar product in $\mathbb{R}^{n}$ is denoted by $\circ$. For $\omega \in C\left(B, \mathbb{R}^{k}\right)$ and $(t, x) \in E_{0} \cup E$ we put $\|\omega\|_{D[t, x]}=\max \{|\omega(\tau, y)|:(\tau, y) \in$ $D[t, x]\}$. The maximum norm in the space $C\left(B, \mathbb{R}^{k}\right)$ will be denoted by $\|\cdot\|_{B}$. Let $C L(B, \mathbb{R})$ denote the set of all linear and continuous real functions defined on $C(B, \mathbb{R})$. The norm in $C L(B, \mathbb{R})$ which is generated by the maximum norm in $C(B, \mathbb{R})$ will be denoted by $\|\cdot\|_{\star}$.

We define a mesh on the set $E_{0} \cup E$ in the following way. Let $\left(h_{0}, h^{\prime}\right)$, $h^{\prime}=\left(h_{1}, \ldots, h_{n}\right)$, stand for steps of the mesh. For $h=\left(h_{0}, h^{\prime}\right)$ and $(r, m) \in$ $\mathbb{Z}^{1+n}$ where $m=\left(m_{1}, \ldots, m_{n}\right)$, we define nodal points in the following way: $t^{(r)}=r h_{0}, x^{(m)}=\left(x_{1}^{\left(m_{1}\right)}, \ldots, x_{n}^{\left(m_{n}\right)}\right)=\left(m_{1} h_{1}, \ldots, m_{n} h_{n}\right)$. Let $\tilde{H}$ denote the set of all $h=\left(h_{0}, h^{\prime}\right)$ such that there are $\left(N_{1}, \ldots, N_{n}\right)=N \in \mathbb{N}^{n}$ and $K_{0} \in \mathbb{Z}$ with $\left(N_{1} h_{1}, \ldots, N_{n} h_{n}\right)=b, K_{0} h_{0}=b_{0}$ and $h^{\prime} \leq M h_{0}$. Define $K \in \mathbb{N}$ by $K h_{0} \leq a<(K+1) h_{0}$. For $h \in \tilde{H}$ we put $\|h\|=h_{0}+h_{1}+\cdots+h_{n}$ and

$$
\mathbb{R}_{h}^{1+n}=\left\{\left(t^{(r)}, x^{(m)}\right):(r, m) \in \mathbb{Z}^{1+n}\right\}, \quad I_{h}=\left\{t^{(r)}: 0 \leq r \leq K\right\} .
$$

Set $E_{0 . h}=E_{0} \cap \mathbb{R}_{h}^{1+n}, E_{h}=E \cap \mathbb{R}_{h}^{1+n}$. For functions $\eta: I_{h} \rightarrow \mathbb{R}^{n}, z: E_{0 . h} \cup$ $E_{h} \rightarrow \mathbb{R}^{k}, u: E_{0 . h} \cup E_{h} \rightarrow M_{k \times n}$, we write $\eta^{(r)}=\eta\left(t^{(r)}\right), z^{(r, m)}=z\left(t^{(r)}, x^{(m)}\right)$, 
$u^{(r, m)}=u\left(t^{(r)}, x^{(m)}\right)$. Let $e_{j}=(0, \ldots, 0,1,0, \ldots, 0) \in \mathbb{R}^{n}$ with 1 in the $j$ th place, $1 \leq j \leq n$. We formulate implicit difference methods of the Euler type for 1.1, 1.2. Write

$$
\begin{aligned}
& E_{i . \epsilon}^{+}=\left\{(t, x) \in E: b_{i}-M_{i} t-\epsilon<x_{i} \leq b_{i}-M_{i} t\right\}, \\
& E_{i . \epsilon}^{-}=\left\{(t, x) \in E: b_{i}+M_{i} t<x_{i} \leq b_{i}+M_{i} t+\epsilon\right\},
\end{aligned}
$$

where $0<\epsilon<b_{i}-M_{i} a$ and $1 \leq i \leq n$.

Assumption $H_{0}[f]$. The function $f: \Omega \rightarrow \mathbb{R}^{k}$ is continuous, satisfies the condition $(V)$ and

1) the partial derivatives

$$
\partial_{x} f=\left[\partial_{x_{j}} f_{i}\right]_{i=1, \ldots, k, j=1, \ldots, n}, \quad \partial_{q} f=\left[\partial_{q_{j}} f_{i}\right]_{i=1, \ldots, k, j=1, \ldots, n}
$$

exist on $\Omega$ and $\partial_{x} f, \partial_{q} f \in C\left(\Omega, M_{k \times n}\right)$,

2) there exist the Fréchet derivatives $\partial_{w} f(P)=\left[\partial_{w_{j}} f_{i}(P)\right]_{i, j=1, \ldots, k}$, and $\partial_{w_{j}} f_{i}(P) \in C L(B, \mathbb{R})$ for $1 \leq i, j \leq k, P \in \Omega$,

3) there is $\epsilon>0$ such that

$$
\begin{array}{ll}
\partial_{q_{i}} f(t, x, w, q) \leq \theta_{[k]} & \text { on } E_{i . \epsilon}^{+} \times C\left(B, \mathbb{R}^{k}\right) \times \mathbb{R}^{n}, \\
\partial_{q_{i}} f(t, x, w, q) \geq \theta_{[k]} & \text { on } E_{i . \epsilon}^{-} \times C\left(B, \mathbb{R}^{k}\right) \times \mathbb{R}^{n},
\end{array}
$$

where $1 \leq i \leq k$ and $\partial_{q_{i}} f=\left(\partial_{q_{i}} f_{1}, \ldots, \partial_{q_{i}} f_{k}\right), \theta_{[k]}=(0, \ldots, 0) \in \mathbb{R}^{k}$.

REMARK 2.1. Suppose that the function $\partial_{q} f: \Omega \rightarrow M_{k \times n}$ satisfies the condition

$$
\left(x_{1} \partial_{q_{1}} f_{i}(P), \ldots, x_{n} \partial_{q_{n}} f_{i}(P)\right) \leq \theta_{[n]}, \quad 1 \leq i \leq k, P \in \Omega,
$$

where $\theta_{[n]}=(0, \ldots, 0) \in \mathbb{R}^{n}$. Then condition 3$)$ of Assumption $H_{0}[f]$ holds true.

Note that if condition 2.1 is satisfied on $[0, a] \times[-b, b] \times C\left(B, \mathbb{R}^{k}\right) \times \mathbb{R}^{k}$ then the natural domain for classical solutions of (1.1), 1.2 is the set $E=$ $[0, a] \times[-b, b]$. Then we put $M=\theta_{[n]}$ in the definition of the Haar pyramid. This property of the initial value problem (1.1), 1.2 follows from the theory of bicharacteristics.

Suppose that Assumption $H_{0}[f]$ is satisfied. Set

$$
H=\left\{h=\left(h_{0}, h^{\prime}\right) \in \tilde{H}: h_{j} \leq \epsilon / 2 \text { for } 1 \leq j \leq n\right\} .
$$

We write $\partial_{x} f_{i}=\left(\partial_{x_{1}} f_{i}, \ldots, \partial_{x_{n}} f_{i}\right), \partial_{w} f_{i}=\left(\partial_{w_{1}} f_{i}, \ldots, \partial_{w_{k}} f_{i}\right)$, where $1 \leq$ $i \leq k$. Set $E_{h}^{\prime}=\left\{\left(t^{(r)}, x^{(m)}\right) \in E_{h}:\left(t^{(r+1)}, x^{(m)}\right) \in E_{h}\right\}$ and $E_{r . h}=$ $\left(E_{0 . h} \cup E_{h}\right) \cap\left(\left[-b_{0}, t^{(r)}\right] \times \mathbb{R}^{n}\right), \quad 0 \leq r \leq K$. We construct a difference problem corresponding to (1.1), (1.2). Unknown functions in a difference system are denoted by $(z, u)$ where $z=\left(z_{1}, \ldots, z_{k}\right), u=\left[u_{i j}\right]_{i=1, \ldots, k, j=1, \ldots, n}$, $u_{i}=\left(u_{i 1}, \ldots, u_{i n}\right), 1 \leq i \leq k$. We denote by $\delta_{0}$ and $\left(\delta_{1}, \ldots, \delta_{n}\right)=\delta$ the difference operators for the variable $t$ and for the spatial variables $\left(x_{1}, \ldots, x_{n}\right)=$ 
$x$ respectively. Write $\delta_{0} u_{i}=\left(\delta_{0} u_{i 1}, \ldots, \delta_{0} u_{i n}\right), \delta z_{i}=\left(\delta_{1} z_{i}, \ldots, \delta_{n} z_{i}\right)$ and $\delta u_{i}=\left[\delta_{j} u_{i s}\right]_{s, j=1, \ldots, n}$ where $1 \leq i \leq k$. Set

$$
\begin{aligned}
& T_{h} u_{(r, m)}=\left[T_{h}\left(u_{i j}\right)_{(r, m)}\right]_{i=1, \ldots, k, j=1, \ldots, n}, \partial_{w} f_{i}(P) \star T_{h} u_{(r, m)} \\
& \quad=\left(\sum_{s=1}^{k} \partial_{w_{s}} f_{i}(P) T_{h}\left(u_{s 1}\right)_{(r, m)}, \ldots, \sum_{s=1}^{k} \partial_{w_{s}} f_{i}(P) T_{h}\left(u_{s n}\right)_{(r, m)}\right), \quad P \in \Omega,
\end{aligned}
$$

and $P^{(r, m)}\left[z, u_{i}\right]=\left(t^{(r)}, x^{(m)}, T_{h} z_{(r, m)}, u_{i}^{(r, m)}\right), 1 \leq i \leq k$. We consider the system of difference equations

$$
\begin{aligned}
\delta_{0} z_{i}^{(r, m)}= & f_{i}\left(P^{(r, m)}\left[z, u_{i}\right]\right) \\
& +\partial_{q} f_{i}\left(P^{(r, m)}\left[z, u_{i}\right]\right) \circ\left(\delta z_{i}^{(r+1, m)}-u_{i}^{(r, m)}\right), \quad 1 \leq i \leq k,
\end{aligned}
$$

and

$$
\begin{aligned}
\delta_{0} u_{i}^{(r, m)}= & \partial_{x} f_{i}\left(P^{(r, m)}\left[z, u_{i}\right]\right)+\partial_{w} f_{i}\left(P^{(r, m)}\left[z, u_{i}\right]\right) \star T_{h} u_{(r, m)} \\
& +\partial_{q} f_{i}\left(P^{(r, m)}\left[z, u_{i}\right]\right) \star\left[\delta u_{i}^{(r+1, m)}\right]^{T}, \quad 1 \leq i \leq k,
\end{aligned}
$$

with the initial conditions

$$
z^{(r, m)}=\varphi_{h}^{(r, m)}, \quad u^{(r, m)}=\psi_{h}^{(r, m)} \quad \text { on } E_{0 . h}
$$

where $\varphi_{h}: E_{0 . h} \rightarrow \mathbb{R}^{k}$ and $\psi_{h}: E_{0 . h} \rightarrow M_{k \times n}$, are given functions. The difference expressions $\delta_{0} z_{i}$ and $\delta_{0} u_{i}$ are defined by

$$
\begin{aligned}
\delta_{0} z_{i}^{(r, m)} & =\frac{1}{h_{0}}\left[z_{i}^{(r+1, m)}-z_{i}^{(r, m)}\right], \\
\delta_{0} u_{i}^{(r, m)} & =\frac{1}{h_{0}}\left[u_{i}^{(r+1, m)}-u_{i}^{(r, m)}\right], \quad 1 \leq i \leq k .
\end{aligned}
$$

The difference operator $\delta$ for the spatial variables is defined in the following way. Suppose that the functions $(z, u)$ are known on the set $E_{r . h}$ where $0 \leq r<K$. We put

(2.6) if $\partial_{q_{j}} f_{i}\left(P^{(r, m)}\left[z, u_{i}\right]\right) \geq 0$ then $\delta_{j} z_{i}^{(r+1, m)}=\frac{1}{h_{j}}\left[z_{i}^{\left(r+1, m+e_{j}\right)}-z_{i}^{(r+1, m)}\right]$

and

$$
\delta_{j} u_{i s}^{(r+1, m)}=\frac{1}{h_{j}}\left[u_{i s}^{\left(r+1, m+e_{j}\right)}-u_{i s}^{(r+1, m)}\right], \quad 1 \leq s \leq n .
$$

Moreover we set

$(2.8)$ if $\partial_{q_{j}} f_{i}\left(P^{(r, m)}\left[z, u_{i}\right]\right)<0$ then $\delta_{j} z_{i}^{(r+1, m)}=\frac{1}{h_{j}}\left[z_{i}^{(r+1, m)}-z_{i}^{\left(r+1, m-e_{j}\right)}\right]$

and

$$
\delta_{j} u_{i s}^{(r+1, m)}=\frac{1}{h_{j}}\left[u_{i s}^{(r+1, m)}-u_{i s}^{\left(r+1, m-e_{j}\right)}\right], \quad 1 \leq s \leq n .
$$


We have $i=1, \ldots, k, j=1, \ldots, n$ in $2.6-2.9$. Note that the difference operators $\delta z_{i}$ and $\delta u_{i}, 1 \leq i \leq k$, are calculated at the point $\left(t^{(r+1)}, x^{(m)}\right)$ in 2.2), 2.3. Then the numbers $z_{i}^{\left(r+1, m+e_{j}\right)}, z_{i}^{\left(r+1, m-e_{j}\right)}$ and the vectors $u_{i}^{\left(r+1, m+e_{j}\right)}, u_{i}^{\left(r+1, m-e_{j}\right)}, 1 \leq j \leq n$, appear on the right hand sides of 2.2 , 2.3). It follows that we have obtained implicit difference schemes.

If we apply classical difference methods to problem $(1.1),(1.2)$ then we approximate derivatives with respect to spatial variables by difference expressions which are calculated by using previous values of approximate solutions. In our method we approximate the spatial derivatives of the unknown function in (1.1) by solving suitable difference equations which are generated by the original problem.

REMARK 2.2. The above construction of an implicit Euler method has the following property: the definition of the difference expressions $\delta z_{i}$ and $\delta u_{i}, 1 \leq i \leq k$, at the point $\left(t^{(r+1)}, x^{(m)}\right)$ depends on the local properties of the functions $\partial_{q} f_{i}, 1 \leq i \leq k$. Note that we construct the Euler type methods and we do not assume that the functions $\left(\operatorname{sign} \partial_{q_{1}} f_{i}, \ldots, \operatorname{sign} \partial_{q_{n}} f_{i}\right)$, $1 \leq i \leq k$, are constant.

REMARK 2.3. Suppose that Assumption $H_{0}[f]$ is satisfied and $z \in$ $C\left(E_{0} \cup E, \mathbb{R}^{k}\right), u \in C\left(E_{0} \cup E, M_{k \times n}\right)$ and $u=\left[u_{i j}\right]_{i=1, \ldots, k, j=1, \ldots, n}, u_{i}=$ $\left(u_{i 1}, \ldots, u_{i n}\right), 1 \leq i \leq k$. Let us consider the Cauchy problem

$$
\eta^{\prime}(t)=-\partial_{q} f_{i}\left(\tau, \eta(\tau), z_{(\tau, \eta(\tau))}, u_{i}(\tau, \eta(\tau))\right), \quad \eta(t)=x,
$$

where $1 \leq i \leq k$ is fixed and $(t, x) \in E$. The solution

$$
g_{i}[z, u](\cdot, t, x)=\left(g_{i 1}[z, u](\cdot, t, x), \ldots, g_{i n}[z, u](\cdot, t, x)\right)
$$

of the above problem is the $i$ th bicharacteristic of system (1.1) corresponding to $(z, u)$. The following property of bicharacteristics is important in our construction of implicit difference schemes for (1.1), (1.2). The difference operators $\left(\delta_{1}, \ldots, \delta_{n}\right)=\delta$ used in this paper satisfy the following conditions:

(i) if the function $g_{i j}[z, u](\cdot, t, x)$ is increasing on $\left[t-\epsilon_{0}, t\right], \epsilon_{0}>0$, then

$$
\begin{aligned}
\delta_{j} z_{i}(t, x) & =\frac{1}{\tau}\left[z_{i}(t, x)-z_{i}\left(t, x-\tau e_{j}\right)\right], \\
\delta_{j} u_{i s}(t, x) & =\frac{1}{\tau}\left[u_{i s}(t, x)-u_{i s}\left(t, x-\tau e_{j}\right)\right], \quad 1 \leq s \leq n,
\end{aligned}
$$

for some $\tau>0$,

(ii) if $g_{i j}[z, u](\cdot, t, x)$ is decreasing on $\left[t-\epsilon_{0}, t\right], \epsilon_{0}>0$, then

$$
\begin{aligned}
\delta_{j} z_{i}(t, x) & =\frac{1}{\tau}\left[z_{i}\left(t, x-\tau e_{j}\right)-z_{i}(t, x)\right], \\
\delta_{j} u_{i s}(t, x) & =\frac{1}{\tau}\left[u_{i s}\left(t, x-\tau e_{j}\right)-u_{i s}(t, x)\right], \quad 1 \leq s \leq n,
\end{aligned}
$$

for some $\tau>0$. 
REMARK 2.4. In our theorem on the convergence of the above difference method we will assume that the initial function $\varphi$ is of class $C^{1}$ on $E_{0}$ and that the functions $\varphi_{h}$ and $\psi_{h}$ are approximations of $\varphi$ and $\partial_{x} \varphi$ respectively.

The difference problem consisting of system (2.2), 2.3) and initial condition (2.4) with the difference operators defined by 2.5 -2.9 is called a generalized implicit Euler method for (1.1), (1.2).

The dfference system (2.2), 2.3 is obtained in the following way. Suppose that Assumption $H_{0}[f]$ is satisfied and that the derivatives $\partial_{x} \varphi=$ $\left[\partial_{x_{j}} \varphi_{i}\right]_{i=1, \ldots, k, j=1, \ldots, n}$ exist on $E_{0}$. We apply the method of quasilinearization to problem (1.1), (1.2). It consists in replacing the nonlinear problem (1.1), 1.2 with the following one. Let $(z, u)$ be unknown functions of $(t, x) \in E$ where $z=\left(z_{1}, \ldots, z_{k}\right), u=\left[u_{i j}\right]_{i=1, \ldots, k, j=1, \ldots, n}, u_{i}=\left(u_{i 1}, \ldots, u_{i n}\right)$ for $1 \leq i \leq k$. Write $U\left[z, u_{i} ; t, x\right]=\left(t, x, z_{(t, x)}, u_{i}(t, x)\right), 1 \leq i \leq k$. We introduce first an additional unknown function $u=\partial_{x} z$ in (1.1). Then we consider the following linearization of (1.1) with respect to the last variable:

$$
\begin{aligned}
\partial_{t} z_{i}(t, x)= & f_{i}\left(U\left[z, u_{i} ; t, x\right]\right) \\
& +\partial_{q} f_{i}\left(U\left[z, u_{i} ; t, x\right]\right) \circ\left(\partial_{x} z_{i}(t, x)-u_{i}(t, x)\right), \quad 1 \leq i \leq k .
\end{aligned}
$$

It follows from (1.1) that $u_{i}, 1 \leq i \leq k$, satisfy the functional differential system

$$
\begin{aligned}
\partial_{t} u_{i}(t, x)= & \partial_{x} f_{i}\left(U\left[z, u_{i} ; t, x\right]\right)+\partial_{w} f_{i}\left(U\left[z, u_{i} ; t, x\right]\right) \star u_{(t, x)} \\
& +\partial_{q} f_{i}\left(U\left[z, u_{i} ; t, x\right]\right) \star\left[\partial_{x} u_{i}(t, x)\right]^{T}, \quad 1 \leq i \leq k .
\end{aligned}
$$

It is natural to consider the following initial condition for the quasilinear system 2.10, 2.11):

$$
z(t, x)=\varphi(t, x), \quad u(t, x)=\partial_{x} \varphi(t, x) \quad \text { on } E_{0} .
$$

We obtain a generalized implicit Euler method for 1.1), 1.2) by a discretization of $2.10-2.12$.

Write $S_{t}=\left\{x \in \mathbb{R}^{n}:(t, x) \in E_{0} \cup E\right\}, t \in\left[-b_{0}, a\right]$, and $U_{x}=\{t \in \mathbb{R}:$ $\left.(t, x) \in E_{0} \cup E\right\}, x \in[-b, b]$. Suppose that $v: E_{0} \cup E \rightarrow \mathbb{R}$ is a classical solution of 1.1), 1.2). We say that $v$ is of class $C^{*}$ if $v(t, \cdot): S_{t} \rightarrow \mathbb{R}^{k}$ is of class $C^{2}$ for every $t \in\left[-b_{0}, a\right]$ and $v(\cdot, x): U_{x} \rightarrow \mathbb{R}^{k}$ is of class $C^{1}$ for every $x \in[-b, b]$.

The initial value problems (1.1), 1.2 and $2.10-(2.12)$ have the following properties ([12, Chapter IV], [22]).

(i) if $v: E_{0} \cup E \rightarrow \mathbb{R}^{k}$ is a classical solution of $11.1, \sqrt{1.2}$ and $v$ is of class $C^{*}$ then the functions $\left(v, \partial_{x} v\right)$ satisfy $2.10-2.12$,

(ii) if $(\tilde{z}, \tilde{u}): E_{0} \cup E \rightarrow \mathbb{R}^{k} \times M_{k \times n}$ is a classical solution of 2.10 2.12 then $\partial_{x} \tilde{z}=\tilde{u}$ and $\tilde{z}$ satisfies (1.1), (1.2) and $\tilde{z}$ is of class $C^{*}$. 
There are different ways of constructing implicit difference schemes for first order partial functional differential equations or systems. We present two such constructions. For simplicity we assume that $k=1$ and that (1.1) does not contain the functional variable. Let us consider the differential equation

$$
\partial_{t} z(t, x)=G\left(t, x, z(t, x), \partial_{x} z(t, x)\right)
$$

with the initial condition 1.2 for $k=1$ and $b_{0}=0$. The difference problem

$$
\begin{aligned}
\delta_{0} z^{(r, m)} & =G\left(t^{(r)}, x^{(m)}, z^{(r+1, m)}, \delta z^{(r+1, m)}\right), \\
z^{(0, m)} & =\varphi_{h}^{(0, m)} \quad \text { for } x^{(m)} \in[-b, b],
\end{aligned}
$$

can be considered as an implicit method for (2.13), (1.2) with $k=1, b_{0}=0$. Note that the difference operators $\left(\delta_{1}, \ldots, \delta_{n}\right)$ and the unknown function $z$ are calculated at the point $\left(t^{(r+1)}, x^{(m)}\right)$.

Let us consider the difference equation

$$
\delta_{0} z^{(r, m)}=G\left(t^{(r)}, x^{(m)}, z^{(r, m)}, \delta z^{(r+1, m)}\right)
$$

with the initial condition 2.15). In this case the unknown function $z$ is calculated at $\left(t^{(r)}, x^{(m)}\right)$ and the difference operators $\left(\delta_{1} z, \ldots, \delta_{n} z\right)$ at $\left(t^{(r+1)}, x^{(m)}\right)$. Thus we have obtained an implicit difference scheme. The paper [4] gives sufficient conditions for the convergence of implicit difference methods of the type 2.16), 2.15) for functional differential equations.

The numerical methods (2.14), 2.15) and implicit difference schemes (2.2) - 2.4 exhibit the following differences. It is clear that a nonlinear differential equation leads to a nonlinear algebraic system (2.14). This system requires iterative schemes for the computation of numerical solutions. In the case when 2.13 is a quasilinear differential equation, the corresponding system of the form (2.14) is also nonlinear.

On the other hand, if we consider the difference scheme $(2.2)-(2.4)$ then we obtain a linear system for the unknowns $\left(z^{(r+1, m)}, u^{(r+1, m)}\right)$. Note that our original problem 1.1, 1.2 is nonlinear.

3. Solutions of implicit Euler schemes. Let us denote by $\left(z_{h}, u_{h}\right): E_{h}$ $\rightarrow \mathbb{R}^{k} \times M_{k \times n}$ the solution of 2.2 2.4 and suppose that the functions $\left(z_{h}, u_{h}\right)$ are calculated on the set $E_{r . h}, 0 \leq r<K$. Our implicit difference methods have the following property: we start with the nonlinear system (1.1), and the existence of a solution $\left(z_{h}, u_{h}\right)$ on $E_{r+1 . h}$ is equivalent to the existence of a solution of the system 2.2), 2.3, which is linear. The difference equations

$$
z_{i}^{(r+1, m)}=h_{0} \partial_{q} f_{i}\left(P^{(r, m)}\left[z, u_{i}\right]\right) \circ \delta z_{i}^{(r+1, m)}, \quad 1 \leq i \leq k,
$$

and

$$
u_{i}^{(r+1, m)}=h_{0} \partial_{q} f_{i}\left(P^{(r, m)}\left[z, u_{i}\right]\right) \star\left[\delta u_{i}^{(r+1, m)}\right]^{T}, \quad 1 \leq i \leq k,
$$


are the principal parts of $(2.2)$ and $(2.3)$ respectively. We prove a lemma on difference inequalities generated by 3.1 , (3.2).

Lemma 3.1. Suppose that

- Assumption $H_{0}[f]$ is satisfied and condition (1.3) holds,

- $h \in H$ and $z_{h} \in \mathcal{F}\left(E_{0 . h} \cup E_{h}, \mathbb{R}^{k}\right), u_{h} \in \mathcal{F}\left(E_{0 . h} \cup E_{h}, M_{k \times n}\right)$ where $z_{h}=$ $\left(z_{h .1}, \ldots, z_{h . k}\right), u_{h}=\left[u_{h . i j}\right]_{i=1, \ldots, k, j=1, \ldots, n}$ and $u_{h . i}=\left(u_{h . i 1}, \ldots, u_{h_{i} n}\right)$, $1 \leq i \leq k$.

(I) If the functions $z_{h}$ and $u_{h}$ satisfy the implicit difference inequalities

$$
z_{h . i}^{(r+1, m)} \leq h_{0} \partial_{q} f_{i}\left(P^{(r, m)}\left[z_{h}, u_{h . i}\right]\right) \circ \delta z_{h . i}^{(r+1, m)}, \quad 1 \leq i \leq k,
$$

and

$$
u_{h . i}^{(r+1, m)} \leq h_{0} \partial_{q} f_{i}\left(P^{(r, m)}\left[z_{h}, u_{h . i}\right]\right) \star\left[\delta u_{h . i}^{(r+1, m)}\right]^{T}, \quad 1 \leq i \leq k,
$$

then

$$
\begin{array}{ll}
z_{h . i}^{(r, m)} \leq 0 & \text { on } E_{h} \text { for } 1 \leq i \leq k, \\
u_{h . i}^{(r, m)} \leq \theta_{[n]} & \text { on } E_{h} \text { for } 1 \leq i \leq k .
\end{array}
$$

(II) If the implicit difference inequalities

$$
z_{h . i}^{(r+1, m)} \geq h_{0} \partial_{q} f_{i}\left(P^{(r, m)}\left[z_{h}, u_{h . i}\right]\right) \circ \delta z_{h . i}^{(r+1, m)}, \quad 1 \leq i \leq k,
$$

and

$$
u_{h . i}^{(r+1, m)} \geq h_{0} \partial_{q} f_{i}\left(P^{(r, m)}\left[z_{h}, u_{h . i}\right]\right) \star\left[\delta u_{h . i}^{(r+1, m)}\right]^{T}, \quad 1 \leq i \leq k,
$$

are satisfied then $z_{h . i}^{(r, m)} \geq 0$ and $u_{h . i}^{(r, m)} \geq \theta_{[n]}$ on $E_{h}$ for $1 \leq i \leq k$.

Proof. Consider case (I). Fix $0 \leq r \leq K-1$. Write

$$
\begin{aligned}
& J_{i .+}^{(r, m)}=\left\{j \in\{1, \ldots, n\}: \partial_{q_{j}} f_{i}\left(P^{(r, m)}\left[z_{h}, u_{h . i}\right]\right) \geq 0\right\}, \\
& J_{i .-}^{(r, m)}=\{1, \ldots, n\} \backslash J_{i .+}^{(r, m)}
\end{aligned}
$$

where $1 \leq i \leq k$. It follows from 3.3 that

$$
\begin{aligned}
z_{h . i}^{(r+1, m)}\left[1+h_{0} \sum_{j=1}^{n} \frac{1}{h_{j}}\left|\partial_{q_{j}} f_{i}\left(P^{(r, m)}\left[z_{h}, u_{h . i}\right]\right)\right|\right] \\
\leq h_{0} \sum_{j \in J_{i .+}^{(r, m)}} \frac{1}{h_{j}} \partial_{q_{j}} f_{i}\left(P^{(r, m)}\left[z_{h}, u_{h . i}\right]\right) z_{h . i}^{\left(r+1, m+e_{j}\right)} \\
\quad-h_{0} \sum_{j \in J_{i .-}^{(r, m)}} \frac{1}{h_{j}} \partial_{q_{j}} f_{i}\left(P^{(r, m)}\left[z_{h}, u_{h . i}\right]\right) z_{h . i}^{\left(r+1, m-e_{j}\right)} .
\end{aligned}
$$

Suppose that $1 \leq i \leq k$ is fixed and $\left(t^{(r+1)}, z^{(\tilde{m})}\right) \in E_{h}$ is such that

$$
z_{h . i}^{(r+1, \tilde{m})} \geq z_{h . i}^{(r+1, m)} \quad \text { for } x^{(m)} \in\left[-b+M t^{(r+1)}, b-M t^{(r+1)}\right] .
$$


If the assertion (3.4) is false then

$$
z_{h . i}^{(r+1, \tilde{m})}>0
$$

From (3.6) we deduce that

$$
\begin{aligned}
z_{h . i}^{(r+1, \tilde{m})}\left[1+h_{0} \sum_{j=1}^{n} \frac{1}{h_{j}}\left|\partial_{q_{j}} f_{i}\left(P^{(r, \tilde{m})}\left[z_{h}, u_{h . i}\right]\right)\right|\right] \\
\leq h_{0} z_{h . i}^{(r+1, \tilde{m})} \sum_{j=1}^{m} \frac{1}{h_{j}}\left|\partial_{q_{j}} f_{i}\left(P^{(r, \tilde{m})}\left[z_{h}, u_{h . i}\right]\right)\right| .
\end{aligned}
$$

Hence $z_{h . i}^{(r+1, \tilde{m})} \leq 0$, which contradicts 3.7 , and the proof of 3.4 is completed by induction. In a similar way we prove (3.5). Case (II) can be treated in the same way. This completes the proof.

Lemma 3.2. If Assumption $H_{0}[f]$ and condition (1.3) are satisfied then there exists exactly one solution $\left(z_{h}, u_{h}\right): E_{0 . h} \cup E_{h} \rightarrow \mathbb{R}^{k} \times M_{k \times n}$ of $[2.2$ (2.4).

Proof. Fix $0 \leq r \leq K-1$ and suppose that the solution $\left(z_{h}, u_{h}\right)$ is known on $E_{r . h}$. Consider the linear system

$$
\begin{aligned}
z_{i}^{(r+1, m)}= & z_{h . i}^{(r, m)}+h_{0} f_{i}\left(P^{(r, m)}\left[z_{h}, u_{h . i}\right]\right) \\
& +h_{0} \partial_{q} f_{i}\left(P^{(r, m)}\left[z_{h}, u_{h . i}\right]\right) \circ\left(\delta z_{i}^{(r+1, m)}-u_{h . i}^{(r+1, m)}\right), \\
u_{i}^{(r+1, m)}= & u_{h . i}^{(r, m)}+h_{0} \partial_{x} f_{i}\left(P^{(r, m)}\left[z_{h}, u_{h . i}\right]\right) \\
& +h_{0} \partial_{w} f_{i}\left(P^{(r, m)}\left[z_{h}, u_{h . i}\right]\right) \star\left(T_{h} u_{h . i}\right)_{(r, m)} \\
& +h_{0} \partial_{q} f_{i}\left(P^{(r, m)}\left[z_{h}, u_{h . i}\right]\right) \star\left[\delta u_{i}^{(r+1, m)}\right]^{T}
\end{aligned}
$$

with unknowns $\left(z_{i}^{(r+1, m)}, u_{i}^{(r+1, m)}\right)$ where $x^{(m)} \in\left[-b+M t^{(r+1)}, b-M t^{(r+1)}\right]$. Suppose that $\left(t^{(r)}, x^{m}\right) \in E_{h}^{\prime}$. It follows from condition 3) of Assumption $H_{0}[f]$ that the expressions $\delta z_{i}^{(r+1, m)}, \delta u_{i}^{(r+1, m)}, 1 \leq i \leq k$, are well defined. We conclude from Lemma 3.1 that the homogeneous system corresponding to (3.8), 3.9) has exactly one zero solution. Then system (3.8), 3.9 has exactly one solution $\left(z_{h . i}^{(r+1, m)}, u_{h . i}^{(r+1, m)}\right)$ where $x^{(m)} \in\left[-b+M t^{(r+1)}, b-M t^{(r+1)}\right]$, and consequently, the functions $\left(z_{h . i}, u_{h . i}\right), 1 \leq i \leq k$, are defined and they are unique on $E_{r+1 . h}$. Since $\left(z_{h}, u_{h}\right)$ are given on $E_{0 . h}$, the proof is completed by induction.

Now we give estimates of solutions to $1.1,21.2$ and $2.2-2.4$. Write

$$
\left\|\partial_{w} f(P)\right\|_{*}=\max \left\{\sum_{j=1}^{k}\left\|\partial_{w_{j}} f_{i}(P)\right\|_{*}: 1 \leq i \leq k\right\}, \quad P \in \Omega .
$$


Assumption $H_{*}[f, \varphi]$. The function $f: \Omega \rightarrow \mathbb{R}^{k}$ satisfies Assumption $H_{0}[f]$ and

1) there is $\tilde{A}>0$ such that $\left\|\partial_{x} f(P)\right\| \leq \tilde{A}$ and $\left\|\partial_{w} f(P)\right\|_{*} \leq \tilde{A}$ for $P=(t, x, w, q) \in \Omega$,

2) for $P \in \Omega$ we have $\left(\left|\partial_{q_{1}} f_{i}(P)\right|, \ldots,\left|\partial q_{n} f_{i}(P)\right|\right) \leq M, 1 \leq i \leq k$,

3) $\varphi: E_{0} \rightarrow \mathbb{R}^{k}$ is of class $C^{1}$ and

$$
\begin{aligned}
& c_{0} \geq \max \left\{\|\varphi(t, x)\|_{\infty}:(t, x) \in E_{0}\right\}, \\
& c_{1} \geq \max \left\{\left\|\partial_{x} \varphi(t, x)\right\|:(t, x) \in E_{0}\right\}
\end{aligned}
$$

and

$$
c_{0} \geq\left\|\varphi_{h}^{(r, m)}\right\|_{\infty}, \quad c_{1} \geq\left\|\psi_{h}^{(r, m)}\right\| \quad \text { for }\left(t^{(r)}, x^{(m)}\right) \in E_{0 . h}, h \in H .
$$

Lemma 3.3. Suppose that Assumption $H_{*}[f, \varphi]$ is satisfied and $v=$ $\left(v_{1}, \ldots, v_{k}\right): E_{0} \cup E \rightarrow \mathbb{R}^{k}$ is a solution to 1.1 , 1.2 and $v$ is of class $C^{*}$ on $E_{0} \cup E$. Then

$$
\|v(t, x)\|_{\infty} \leq \bar{\alpha}_{0}(t), \quad\left\|\partial_{x} v(t, x)\right\| \leq \bar{\alpha}_{1}(t) \quad \text { for }(t, x) \in E
$$

where

$$
\begin{aligned}
\bar{\alpha}_{0}(t)= & c_{0} e^{\tilde{A} t}+\frac{\tilde{c}}{\tilde{A}}\left(e^{\tilde{A} t}-1\right)+2\|M\| c_{1} t e^{\tilde{A} t} \\
& +\frac{2\|M\|}{\tilde{A}}\left[1+\tilde{A} t e^{\tilde{A} t}-e^{\tilde{A} t}\right], \\
\bar{\alpha}_{1}(t)= & \left(c_{1}+1\right) e^{\tilde{A} t}-1
\end{aligned}
$$

and

$$
\tilde{c}=\max \left\{\left\|f\left(t, x, \mathbf{O}, \theta_{[n]}\right)\right\|_{\infty}:(t, x) \in E\right\}
$$

where $\mathbf{O} \in C\left(B, \mathbb{R}^{k}\right)$ is given by $\mathbf{O}(\tau, y)=0$ for $(\tau, y) \in B$.

Proof. Let us denote by $g_{i}[z, u](\cdot, t, x)$ the $i$ th bicharacteristic of 1.1 corresponding to $(z, u)$. It follows that the functions $\left(v, \partial_{x} v_{i}\right), 1 \leq i \leq k$, satisfy the integral functional system

$$
\begin{aligned}
z_{i}(t, x)= & \varphi\left(0, g_{i}[z, u](0, t, x)\right)+\int_{0}^{t} f_{i}\left(P_{i}[z, u](\tau, t, x)\right) d \tau \\
& -\int_{0}^{t} \partial_{q} f_{i}\left(P_{i}[z, u](\tau, t, x)\right) \circ u_{i}\left(\tau, g_{i}[z, u](\tau, t, x)\right) d \tau, \\
u_{i}(t, x)= & \partial_{x} \varphi_{i}\left(0, g_{i}[z, u](0, t, x)\right)+\int_{0}^{t} \partial_{x} f_{i}\left(P_{i}[z, u](\tau, t, x)\right) d \tau \\
& +\int_{0}^{t} \partial_{w} f_{i}\left(P_{i}[z, u](\tau, t, x)\right) \star u_{\left(\tau, g_{i}[z, u](\tau, t, x)\right)} d \tau, \quad 1 \leq i \leq k,
\end{aligned}
$$


and the initial conditions $z(t, x)=\varphi(t, x), u(t, x)=\partial_{x} \varphi(t, x)$ on $E_{0}$ where $P_{i}[z, u](\tau, t, x)=\left(\tau, g_{i}[z, u](\tau, t, x), z_{\left(\tau, g_{i}[z, u](\tau, t, x)\right)}, u_{i}\left(\tau, g_{i}[z, u](\tau, t, x)\right)\right)$, and $1 \leq i \leq k$. Write

$$
\begin{aligned}
& \tilde{\alpha}_{0}(t)=\max \left\{\|v(\tau, y)\|_{\infty}:(\tau, y) \in E_{0} \cup E, \tau \leq t\right\}, \\
& \tilde{\alpha}_{1}(t)=\max \left\{\left\|\partial_{x} v(\tau, y)\right\|:(\tau, y) \in E_{0} \cup E, \tau \leq t\right\},
\end{aligned}
$$

where $t \in[0, a)$. It follows that the functions $\left(\tilde{\alpha}_{0}, \tilde{\alpha}_{1}\right)$ satisfy the integral inequalities

$$
\begin{aligned}
& \tilde{\alpha}_{0}(t) \leq c_{0}+\tilde{c} t+\int_{0}^{t}\left[\tilde{A} \tilde{\alpha}_{0}(\tau)+2\|M\| \tilde{\alpha}_{1}(\tau)\right] d \tau \\
& \tilde{\alpha}_{1}(t) \leq c_{1}+\tilde{A} t+\tilde{A} \int_{0}^{t} \tilde{\alpha}_{1}(\tau) d \tau .
\end{aligned}
$$

Hence the functions $\left(\tilde{\alpha}_{0}, \tilde{\alpha}_{1}\right)$ satisfy the equations corresponding to the above integral inequalities. Thus the assertion $\sqrt{3.10}$ follows.

Assumption $H\left[T_{h}\right]$. The function $T_{h}: \mathcal{F}\left(E_{0 . h} \cup E_{h}, \mathbb{R}^{k}\right) \rightarrow C\left(E_{0} \cup E, \mathbb{R}^{k}\right)$ is such that

1) for $w, \tilde{w}: E_{0 . h} \cup E_{h} \rightarrow \mathbb{R}^{k}$ we have $\left\|T_{h} w-T_{h} \tilde{w}\right\|_{t^{(r)}} \leq\|w-\tilde{w}\|_{r . h}$, $0 \leq r \leq K$

2) for each $w: E_{0} \cup E \rightarrow \mathbb{R}^{k}$ of class $C^{1}$ there is $\gamma_{*}: H \rightarrow \mathbb{R}_{+}$such that

$$
\left\|w-T_{h} w_{h}\right\|_{t} \leq \gamma_{*}(h), \quad 0 \leq t \leq a, \quad \lim _{h \rightarrow 0} \gamma_{*}(h)=0
$$

where $w_{h}=\left.w\right|_{E_{h .0} \cup E_{h}}$,

3) if $\mathbf{O}_{h} \in \mathcal{F}\left(E_{0 . h} \cup E_{h}, \mathbb{R}\right)$ is given by $\mathbf{O}_{h}(t, x)=0$ for $(t, x) \in E_{0 . h} \cup E_{h}$ then $\left(T_{h} \mathbf{O}_{h}\right)(t, x)=0$ for $(t, x) \in E_{0} \cup E$.

REMARK 3.1. The above condition 1) states that $T_{h}$ satisfies the Lipschitz condition with constant $L=1$. The meaning of condition 2) is that $T_{h} w_{h}$ is an approximation of $w$ and the approximation error is estimated by $\gamma_{*}(h)$. An example of $T_{h}$ which satisfies the above conditions can be found in [12, Chapter 3].

Lemma 3.4. Suppose that Assumption $H_{*}[f, \varphi]$ and condition (1.3) are satisfied and $\left(z_{h}, u_{h}\right): E_{0 . h} \cup E_{h} \rightarrow \mathbb{R}^{k} \times M_{k \times n}$ is a solution to 2.2 2.4 with $\delta_{0}$ and $\delta$ defined by (2.5)-(2.9). Then

$$
\left\|z_{h}^{(r, m)}\right\|_{\infty} \leq \bar{\alpha}_{0}^{(r)}, \quad\left\|u_{h}^{(r, m)}\right\| \leq \bar{\alpha}_{1}^{(r)} \quad \text { on } E_{h}
$$

where $\bar{\alpha}_{0}, \bar{\alpha}_{1}$ are given by (3.11), 3.12). 
Proof. Write

$$
\begin{aligned}
& \alpha_{h .0}^{(r)}=\max \left\{\left\|z_{h}^{(s, m)}\right\|_{\infty}:\left(t^{(s)}, x^{(m)}\right) \in E_{r . h}\right\}, \\
& \alpha_{h .1}^{(r)}=\max \left\{\left\|u_{h}^{(s, m)}\right\|:\left(t^{(s)}, x^{(m)}\right) \in E_{r . h}\right\}
\end{aligned}
$$

where $0 \leq r \leq K$. Easy computations show that the functions $\left(\alpha_{h .0}, \alpha_{h .1}\right)$ satisfy the difference inequalities

$$
\begin{aligned}
& \frac{1}{h_{0}}\left[\alpha_{h .0}^{(r+1)}-\alpha_{h .0}^{(r)}\right] \leq \tilde{c}+\tilde{A} \alpha_{h .0}^{(r)}+2\|M\| \alpha_{h .1}^{(r)}, \\
& \frac{1}{h_{0}}\left[\alpha_{h .1}^{(r+1)}-\alpha_{h .1}^{(r)}\right] \leq \tilde{A}\left[1+\alpha_{h .1}^{(r)}\right], \quad 0 \leq r \leq K-1 .
\end{aligned}
$$

The functions $\left(\bar{\alpha}_{0}, \bar{\alpha}_{1}\right)$ satisfy the differential equations

$$
y_{0}^{\prime}(t)=\tilde{c}+\tilde{A} y_{0}(t)+2\|M\| y_{1}(t), \quad y_{1}^{\prime}(t)=\tilde{A}+\tilde{A} y_{1}(t) .
$$

and $\bar{\alpha}_{0}^{(0)}=\bar{\alpha}_{h .0}^{(0)}, \bar{\alpha}_{1}^{(0)}=\bar{\alpha}_{h .1}^{(0)}$. Thus the assertion 3.13 follows.

4. Convergence of the generalized implicit Euler method. Suppose that Assumption $H_{*}[f, \varphi]$ is satisfied. Write $d=\bar{\alpha}_{0}(a), \tilde{d}=\bar{\alpha}_{1}(a)$ where $\left(\bar{\alpha}_{0}, \bar{\alpha}_{1}\right)$ are given by $3.11,3.12$ and $\Omega[d, \tilde{d}]=E \times K_{C\left(B, \mathbb{R}^{k}\right)}[d] \times K_{\mathbb{R}^{n}}[\tilde{d}]$ where

$K_{C\left(B, \mathbb{R}^{k}\right)}[d]=\left\{w \in C\left(B, \mathbb{R}^{k}\right):\|w\|_{B} \leq d\right\}, \quad K_{\mathbb{R}^{n}}[\tilde{d}]=\left\{q \in \mathbb{R}^{n}:\|q\| \leq \tilde{d}\right\}$. We will need the following assumptions on $f$.

Assumption $H[f, \varphi]$. The functions $f: \Omega \rightarrow \mathbb{R}^{k}$ and $\varphi: E_{0} \rightarrow \mathbb{R}^{k}$ satisfy Assumption $H_{*}[f, \varphi]$ and

1) there is $\sigma \in C\left([0, a] \times \mathbb{R}_{+}, \mathbb{R}_{+}\right)$such that

- $\sigma$ is continuous and nondecreasing with respect to both variables,

- $\sigma(t, 0)=0$ for $t \in[0, a]$ and for each $c \geq 1$ the maximal solution of the Cauchy problem

$$
\eta^{\prime}(t)=c[\eta(t)+\sigma(t, \eta(t))], \quad \eta(0)=0,
$$

is $\bar{\eta}(t)=0$ for $t \in[0, a]$,

2) the terms

$$
\begin{gathered}
\left\|\partial_{x} f_{i}(t, x, w, q)-\partial_{x} f_{i}(t, x, \bar{w}, \bar{q})\right\|,\left\|\partial_{q} f_{i}(t, x, w, q)-\partial_{q} f_{i}(t, x, \bar{w}, \bar{q})\right\|, \\
\left\|\partial_{w} f_{i}(t, x, w, q)-\partial_{w} f_{i}(t, x, \bar{w}, \bar{q})\right\|
\end{gathered}
$$

are estimated by $\sigma\left(t,\|w-\bar{w}\|_{B}+\|q-\bar{q}\|\right)$ for $1 \leq i \leq k$ on $\Omega[d, \tilde{d}]$.

REMARK 4.1. Theorems on difference methods presented in [4, [13] have the following property: it is assumed that the functions $\partial_{x} f, \partial_{q} f, \partial_{w} f$ satisfy nonlinear conditions of the Perron type with respect to $(w, q)$ and the estimates are global with respect to $(w, q)$. Note that we have assumed estimates 
of the Perron type with respect to $(w, q)$, local with respect to $(w, q)$. It is clear that there are differential systems with deviated variables and integral differential systems such that condition 2) holds and global estimates of the Perron type with respect to $(w, q)$ are not satisfied.

Now we formulate the main result of the paper.

TheOREM 4.1. Suppose that Assumptions $H[f, \varphi]$ and $H\left[T_{h}\right]$ are satisfied and

1) $\varphi \in C\left(E_{0}, \mathbb{R}^{k}\right)$ and $\varphi(t, \cdot):[-b, b] \rightarrow \mathbb{R}^{k}$ is of class $C^{2}$ for every $t \in$ $\left[-b_{0}, 0\right]$ and $\varphi(\cdot, x):\left[-b_{0}, 0\right] \rightarrow \mathbb{R}^{k}$ is of class $C^{1}$ for every $x \in[-b, b]$,

2) $v: E_{0} \cup E \rightarrow \mathbb{R}^{k}$ is the solution to (1.1), 1.2 and $v$ is of class $C^{*}$ on $E_{0} \cup E$,

3) $\left(z_{h}, u_{h}\right): E_{0 . h} \cup E_{h} \rightarrow \mathbb{R}^{k} \times M_{k \times n}$ is the solution to 2.2 - 2.4 with $\delta_{0}, \delta$ defined by 2.5 - 2.9) and there is $\alpha_{0}: H \rightarrow \mathbb{R}_{+}$such that

$$
\left\|\varphi^{(r, m)}-\varphi_{h}^{(r, m)}\right\|+\left\|\partial_{x} \varphi^{(r, m)}-\psi_{h}^{(r, m)}\right\| \leq \alpha_{0}(h) \quad \text { on } E_{0 . h}
$$

and $\lim _{h \rightarrow 0} \alpha_{0}(h)=0$.

Then there exists $\alpha: H \rightarrow \mathbb{R}_{+}$such that

$$
\left\|v_{h}-z_{h}\right\|_{r . h}+\left\|\partial_{x} v_{h}-u_{h}\right\|_{r . h} \leq \alpha_{0}(h) \quad \text { for } 0 \leq r \leq K,
$$

and $\lim _{h \rightarrow 0} \alpha(h)=0$ where $v_{h}$ and $\partial_{x} v_{h}$ are the restrictions of $v$ and $\partial_{x} v$, respectively, to the set $E_{0 . h} \cup E_{h}$.

Proof. Write $\chi=\partial_{x} v, \chi=\left[\chi_{i j}\right]_{i=1, \ldots, k, j=1, \ldots, n}, \chi_{i}=\left(\chi_{i 1}, \ldots, \chi_{i n}\right), 1 \leq$ $i \leq k$, and

$$
\begin{aligned}
& \chi_{h}=\left.\chi\right|_{E_{0 . h} \cup E_{h}}, \\
& \chi_{h}=\left[\chi_{h . i j}\right]_{i=1, \ldots, k, j=1, \ldots, n}, \chi_{h . i}=\left(\chi_{h . i 1}, \ldots, \chi_{h . i n}\right), \quad 1 \leq i \leq k .
\end{aligned}
$$

Then $(v, \chi)$ satisfy the quasilinear system (2.10), (2.11) and the initial condition 2.12. Consider the functions $\xi_{h}: E_{0 . h} \cup E_{h} \rightarrow \mathbb{R}^{k}$ and $\lambda_{h}: E_{0 . h} \cup$ $E_{h} \rightarrow M_{k \times n}$ defined by $\xi_{h}=v_{h}-z_{h}, \xi_{h}=\left(\xi_{h .1}, \ldots, \xi_{h . k}\right), \lambda_{h}=\chi_{h}-u_{h}$, $\lambda_{h}=\left[\lambda_{h . i j}\right]_{i=1, \ldots, k, j=1, \ldots, n}$ and $\lambda_{h . i}=\left(\lambda_{h . i 1}, \ldots, \lambda_{h . i n}\right), 1 \leq i \leq k$. Let $\omega_{h .0}, \omega_{h .1}: I_{h} \rightarrow \mathbb{R}_{+}$be given by

$$
\begin{aligned}
& \omega_{h .0}^{(r)}=\max \left\{\left\|\xi_{h}^{(i, m)}\right\|_{\infty}:\left(t^{(i)}, x^{(m)}\right) \in E_{r . h}\right\}, \\
& \omega_{h .1}^{(r)}=\max \left\{\left\|\lambda_{h}^{(i, m)}\right\|_{\infty}:\left(t^{(i)}, x^{(m)}\right) \in E_{r . h}\right\}
\end{aligned}
$$

and $\omega_{h}=\omega_{h .0}+\omega_{h .1}$. We will write a difference inequality for $\omega_{h}$. We first examine $\omega_{h .0}$. Set $Q^{(r, m)}\left[v, \chi_{i}\right]=\left(t^{(r)}, x^{(m)}, v_{\left(t^{r)}, x^{(m)}\right.}, \chi_{i}^{(r, m)}\right), 1 \leq i \leq k$. Let the functions $\Gamma_{h}, \Lambda_{h}: E_{h}^{\prime} \rightarrow \mathbb{R}^{k}, \Gamma_{h}=\left(\Gamma_{h .1}, \ldots, \Gamma_{h . k}\right), \Lambda_{h}=\left(\Lambda_{h .1}, \ldots, \Lambda_{h . k}\right)$, be defined by

$$
\Gamma_{h . i}^{(r, m)}=\delta_{0} v_{h . i}^{(r, m)}-\partial_{t} v_{i}^{(r, m)}+\partial_{q} f_{i}\left(Q^{(r, m)}\left[v, \chi_{i}\right]\right) \circ\left[\partial_{x} v_{i}^{(r, m)}-\delta v_{h . i}^{(r+1, m)}\right]
$$


and

$$
\begin{aligned}
\Lambda_{h . i}^{(r, m)}= & f_{i}\left(Q^{(r, m)}\left[v, \chi_{i}\right]\right)-f_{i}\left(P^{(r, m)}\left[z_{h}, u_{h . i}\right)\right. \\
& -\partial_{q} f_{i}\left(Q^{(r, m)}\left[v, \chi_{i}\right]\right) \circ \chi_{i}^{(r, m)}+\partial_{q} f_{i}\left(P^{(r, m)}\left[z_{h}, u_{h . i}\right]\right) \circ u_{h . i}^{(r, m)} \\
& +\left[\partial_{q} f_{i}\left(Q^{(r, m)}\left[v, \chi_{i}\right]\right)-\partial_{q} f_{i}\left(P^{(r, m)}\left[z_{h}, u_{h}\right]\right)\right] \circ \delta v_{h . i}^{(r+1, m)}
\end{aligned}
$$

where $1 \leq i \leq k$. It follows from 2.2, 2.10) that $\xi_{h}$ satisfies the difference equations

$$
\delta_{0} \xi_{h . i}^{(r, m)}=\partial_{q} f_{i}\left(P^{(r, m)}\left[z_{h}, u_{h . i}\right]\right) \circ \delta \xi_{h . i}^{(r+1, m)}+\Lambda_{h . i}^{(r, m)}+\Gamma_{h . i}^{(r, m)}, 1 \leq i \leq k .
$$

We conclude from $2.5-2.6$ that the above relations are equivalent to

$$
\begin{aligned}
\xi_{h . i}^{(r+1, m)}[1 & \left.+h_{0} \sum_{j=1}^{n} \frac{1}{h_{j}}\left|\partial_{q_{j}} f_{i}\left(P^{(r, m)}\left[z_{h}, u_{h . i}\right]\right)\right|\right] \\
= & \xi_{h . i}^{(r, m)}+h_{0} \sum_{j \in J_{i .+}^{(r, m)}} \frac{1}{h_{j}} \partial_{q_{j}} f_{i}\left(P^{(r, m)}\left[z_{h}, u_{h . i}\right]\right) \xi_{h . i}^{\left(r+1, m+e_{j}\right)} \\
& -h_{0} \sum_{j \in J_{i .-}^{(r, m)}} \frac{1}{h_{j}} \partial_{q_{j}} f_{i}\left(P^{(r, m)}\left[z_{h}, u_{h . i}\right]\right) \xi_{h . i}^{\left(r+1, m-e_{j}\right)} \\
& +h_{0}\left[\Gamma_{h . i}^{(r, m)}+\Lambda_{h . i}^{(r, m)}\right], \quad 1 \leq i \leq k .
\end{aligned}
$$

It follows easily that there is $\gamma_{0}: H \rightarrow \mathbb{R}_{+}$such that

$$
\left\|\Gamma_{h}^{(r, m)}\right\| \leq \gamma_{0}(h) \quad \text { on } E_{h}^{\prime}, \quad \lim _{h \rightarrow 0} \gamma_{0}(h)=0 .
$$

We conclude from Lemma 3.3, Lemma 3.4 and Assumption $H\left[T_{h}\right]$ that

$$
\begin{aligned}
\left\|v_{\left(t^{(r)}, x^{(m)}\right)}\right\|_{D\left[t^{(r)}, x^{(m)}\right]} & \leq d, \quad\left\|T_{h}\left(z_{h}\right)_{(r, m)}\right\|_{D\left[t^{(r)}, x^{(m)}\right]} \leq d, \\
\left\|\chi_{i}^{(r, m)}\right\| & \leq \tilde{d}, \quad\left\|u_{h . i}^{(r, m)}\right\| \leq \tilde{d}
\end{aligned}
$$

where $\left(t^{(r)}, x^{(m)}\right) \in E_{h}^{\prime}$. Let $\tilde{a}=\max \{d, \tilde{d}\}$. Write $A=\max \{\tilde{A},\|M\|\}$. According to Assumption $H[f, \varphi]$ we have

$$
\left\|f_{i}\left(Q^{(r, m)}\left[v, \chi_{i}\right]\right)-f_{i}\left(P^{(r, m)}\left[z_{h}, u_{h . i}\right]\right)\right\| \leq A \omega_{h}^{(r)} \text { on } E_{h}^{\prime} \text { for } 1 \leq i \leq k,
$$

and

$$
\left\|\partial_{q} f_{i}\left(Q^{(r, m)}\left[v, \chi_{i}\right]\right)-\partial_{q} f_{i}\left(P^{(r, m)}\left[z_{h}, u_{h . i}\right]\right)\right\| \leq \sigma\left(t^{(r)}, \omega_{h}^{(r)}\right)
$$

on $E_{h}^{\prime}$ for $1 \leq i \leq k$. Then we have

$$
\left|\Lambda_{h . i}^{(r, m)}\right| \leq 2 A \omega_{h}^{(r)}+2 \tilde{a} \sigma\left(t^{(r)}, \omega_{h}^{(r)}\right) .
$$


We see at once that

$$
\begin{aligned}
\sum_{j \in J_{i}^{+}[r, m]} \frac{1}{h_{j}} \partial_{q_{j}} f_{i}\left(P^{(r, m)}\left[z_{h}, u_{h . i}\right]\right)\left|\xi_{h . i}^{\left(r+1, m+e_{j}\right)}\right| \\
-\sum_{j \in J_{i}^{-}[r, m]} \frac{1}{h_{j}} \partial_{q_{j}} f_{i}\left(P^{(r, m)}\left[z_{h}, u_{h . i}\right]\right)\left|\xi_{h . i}^{\left(r+1, m-e_{j}\right)}\right| \\
\leq \omega_{h .0}^{(r+1)} \sum_{j=1}^{n} \frac{1}{h_{j}}\left|\partial_{q_{j}} f_{i}\left(P^{(r, m)}\left[z_{h}, u_{h . i}\right]\right)\right| .
\end{aligned}
$$

From 4.6), 4.8, 4.10, 4.11) we get

$$
\omega_{h .0}^{(r+1)} \leq \omega_{h .0}^{(r)}+2 h_{0} A \omega_{h}^{(r)}+2 h_{0} \tilde{a} \sigma\left(t^{(r)}, \omega_{h}^{(r)}\right)+h_{0} \gamma_{0}(h)
$$

for $0 \leq r \leq K-1$. Now we write a difference inequality for $\omega_{h .1}$. Let the functions $U_{h . i}, Z_{h . i}: E_{h}^{\prime} \rightarrow \mathbb{R}^{n}, 1 \leq i \leq k$, be defined by

$$
\begin{aligned}
U_{h . i}^{(r, m)}= & \delta_{0} \chi_{h . i}^{(r, m)}-\partial_{t} \chi_{i}^{(r, m)} \\
& +\partial_{q} f_{i}\left(Q^{(r, m)}\left[v, \chi_{i}\right]\right) \star\left[\partial_{x} \chi_{i}^{(r, m)}-\delta \chi_{h . i}^{(r+1, m)}\right]^{T}
\end{aligned}
$$

and

$$
\begin{aligned}
& Z_{h . i}^{(r, m)}=\partial_{x} f_{i}\left(Q^{(r, m)}\left[v, \chi_{i}\right]\right)-\partial_{x} f_{i}\left(P^{(r, m)}\left[z_{h}, u_{h . i}\right]\right) \\
+ & \partial_{w} f_{i}\left(Q^{(r, m)}\left[v, \chi_{i}\right]\right) \star\left(\chi_{i}\right)_{(r, m)}-\partial_{w} f_{i}\left(P^{(r, m)}\left[z_{h}, u_{h . i}\right]\right) \star\left(T_{h} u_{h . i}\right)_{(r, m)} \\
+ & {\left[\partial_{q} f_{i}\left(Q^{(r, m)}\left[v, \chi_{i}\right]\right)-\partial_{q} f_{i}\left(P^{(r, m)}\left[z_{h}, u_{h . i}\right]\right)\right] \star\left[\delta \chi_{h . i}^{(r+1, m)}\right]^{T} . }
\end{aligned}
$$

Then the functions $\lambda_{h . i}, 1 \leq i \leq k$, satisfy the difference equations

$$
\delta_{0} \lambda_{h . i}^{(r, m)}=\partial_{q} f_{i}\left(P^{(r, m)}\left[z_{h}, u_{h . i}\right]\right) \star\left[\delta \lambda_{h . i}^{(r+1, m)}\right]^{T}+U_{h . i}^{(r, m)}+Z_{h . i}^{(r, m)} .
$$

It follows easily that the above relations are equivalent to

$$
\begin{aligned}
& \lambda_{h . i}^{(r+1, m)}\left[1+h_{0} \sum_{j=1}^{n} \frac{1}{h_{j}}\left|\partial_{q_{j}} f_{i}\left(P^{(r, m)}\left[z_{h}, u_{h . i}\right]\right)\right|\right] \\
= & \lambda_{h . i}^{(r, m)}+h_{0} \sum_{j \in J_{i .+}^{(r, m)}} \frac{1}{h_{j}} \partial_{q_{j}} f_{i}\left(P^{(r, m)}\left[z_{h}, u_{h . i}\right]\right) \lambda_{h . i}^{\left(r+1, m+e_{j}\right)} \\
- & h_{0} \sum_{j \in J_{i .-}^{(r, m)}} \frac{1}{h_{j}} \partial_{q_{j}} f_{i}\left(P^{(r, m)}\left[z_{h}, u_{h . i}\right]\right) \lambda_{h . i}^{\left(r+1, m-e_{j}\right)}+h_{0}\left[U_{h . i}^{(r, m)}+Z_{h . i}^{(r, m)}\right] .
\end{aligned}
$$

It is clear that there is $\gamma: H \rightarrow \mathbb{R}_{+}$such that

$$
\left\|U_{h . i}^{(r, m)}\right\| \leq \gamma(h) \quad \text { on } E_{h}^{\prime} \text { for } 1 \leq i \leq k \quad \text { and } \quad \lim _{h \rightarrow 0} \gamma(h)=0 .
$$

The estimates analogous to 4.9 can be obtained for $\partial_{x} f_{i}$ and $\partial_{w} f_{i}$. It follows 
from 4.14 and Assumptions $H[f, \varphi], H\left[T_{h}\right]$ that

$$
\left\|Z_{h}^{(r, m)}\right\| \leq(1+2 \tilde{a}) \sigma\left(t^{(r)}, \omega_{h}^{(r)}\right)+A \omega_{h}^{(r)} .
$$

It is easy to see that

$$
\begin{gathered}
\sum_{j \in J_{i}^{+}[r, m]} \frac{1}{h_{j}} \partial_{q_{j}} f_{i}\left(P^{(r, m)}\left[z_{h}, u_{h . i}\right]\right)\left\|\lambda_{h . i}^{\left(r+1, m+e_{j}\right)}\right\| \\
-\sum_{j \in J_{i}^{-}[r, m]} \frac{1}{h_{j}} \partial_{q_{j}} f_{i}\left(P^{(r, m)}\left[z_{h}, u_{h . i}\right]\right)\left\|\lambda_{h . i}^{\left(r+1, m-e_{j}\right)}\right\| \\
\leq \omega_{1 . h}^{(r+1)} \sum_{j=1}^{n} \frac{1}{h_{j}}\left|\partial_{q_{j}} f_{i}\left(P^{(r, m)}\left[z_{h}, u_{h . i}\right]\right)\right| .
\end{gathered}
$$

We deduce from 4.15, 4.17, 4.18 that

$$
\omega_{h .1}^{(r+1)} \leq \omega_{h .1}^{(r)}+h_{0} A \omega_{h}^{(r)}+h_{0}(1+2 \tilde{a}) \sigma\left(t^{(r)}, \omega_{h}^{(r)}\right)+h_{0} \gamma(h)
$$

for $0 \leq r \leq K-1$. Adding inequalities 4.12, 4.19) we get

$$
\omega_{h}^{(r+1)} \leq \omega_{h}^{(r)}+h_{0}(1+4 \tilde{a}) \sigma\left(t^{(r)}, \omega_{h}^{(r)}\right)+3 h_{0} A \omega_{h}^{(r)}+h_{0}\left(\gamma_{0}(h)+\gamma(h)\right)
$$

for $0 \leq r \leq K-1$. Consider the Cauchy problem

$$
\omega^{\prime}(t)=3 A \omega(t)+(1+4 \tilde{a}) \sigma(t, \omega(t))+\left(\gamma_{0}(h)+\gamma(h)\right), \quad \omega(0)=\alpha_{0}(h) .
$$

From Assumption $H[f, \varphi]$ we know that there is $\epsilon_{0}>0$ such that for $\|h\|<\epsilon_{0}$ there exists a maximal solution $\eta_{h}:[0, a] \rightarrow \mathbb{R}_{+}$of 4.21 and $\lim _{h \rightarrow 0} \eta_{h}(t)=0$ uniformly on $[0, a]$. The following recurrent inequality is satisfied:

$$
\eta_{h}^{(r+1)} \geq \eta_{h}^{(r)}+3 h_{0} A \eta_{h}^{(r)}+h_{0}(1+4 \tilde{a}) \sigma\left(t^{(r)}, \eta_{h}^{(r)}\right)+h_{0}\left(\gamma_{0}(h)+\gamma(h)\right),
$$

where $0 \leq r \leq K-1$. Since $\omega_{h}^{(r)} \leq \eta_{h}^{(r)}$, from the above inequality and 4.20 we see that $\omega_{h}^{(r)} \leq \eta_{h}^{(r)}$ for $0 \leq r \leq K$. We obtain the estimate 4.2 for $\alpha(h)=\eta_{h}(a)$. This completes the proof.

REMARK 4.2. Note that the (CFL) conditions are not assumed in Theorem 4.1.

5. Comments and examples. Suppose that all the assumptions of Theorem 4.1 are satisfied with $\sigma(t, p)=L p$ on $[0, a] \times \mathbb{R}_{+}$where $L>0$. Then

$$
\left\|v_{h}-z_{h}\right\|_{r . h}+\left\|\partial_{x} v_{h}-u_{h}\right\|_{r . h} \leq \tilde{\alpha}(h), \quad 0 \leq r \leq K
$$

where

$$
\tilde{\alpha}(h)=\alpha_{0}(h) e^{\tilde{L} t}+\frac{1}{\tilde{L}}\left(\gamma_{0}(h)+\gamma(h)\right)\left(e^{\tilde{L} t}-1\right)
$$


and $\tilde{L}=3 A+L(1+4 \tilde{a})$. Inequality $(5.1)$ is obtained by solving the Cauchy problem 4.21 with $\sigma(t, p)=L p$.

Lemma 5.1. Suppose that

1) Assumption $H[f, \varphi]$ is satisfied with $\sigma(t, p)=L p$ on $[0, a] \times \mathbb{R}_{+}$where $L>0$,

2) the operator $T_{h}: \mathcal{F}\left(E_{0 . h} \cup E_{h}, \mathbb{R}^{k}\right) \rightarrow C\left(E_{0} \cup E, \mathbb{R}\right)$ is such that

- for $w, \tilde{w} \in \mathcal{F}\left(E_{0 . h} \cup E_{h}, \mathbb{R}^{k}\right)$ we have $\left\|T_{h} w-T_{h} \tilde{w}\right\|_{t^{(r)}} \leq\|w-\tilde{w}\|_{r . h}$, $0 \leq r \leq K$,

- for each $w: E_{0} \cup E \rightarrow \mathbb{R}^{k}$ of class $C^{2}$ there is $C_{*} \in \mathbb{R}_{+}$such that $\left\|w-T_{h} w_{h}\right\|_{t} \leq C_{*}\|h\|, 0 \leq t \leq a$, where $w_{h}=\left.w\right|_{E_{0 . h} \cup E_{h}}$,

3) $\varphi: E_{0} \rightarrow \mathbb{R}^{k}$ is of class $C^{3}$ and $v: E_{0} \cup E \rightarrow \mathbb{R}^{k}$ is the solution of (1.1), 1.2 and $v$ is of class $C^{3}$ on $E_{0} \cup E$,

4) condition 2) of Theorem 4.1 is satisfied.

Then there is $\tilde{C} \in \mathbb{R}_{+}$such that

$$
\left\|v_{h}-z_{h}\right\|_{r . h}+\left\|\partial_{x} v_{h}-u_{h}\right\|_{r . h} \leq C_{0} \alpha_{0}(h)+\tilde{C}\|h\|, \quad 0 \leq r \leq K,
$$

where $C_{0}=\exp [(3 A+L(1+4 \tilde{a})) a]$.

Proof. There are $c_{0}, c \in \mathbb{R}_{+}$such that conditions (4.7) and (4.16) are satisfied with $\gamma_{0}(h)=c_{0}\|h\|$ and $\gamma(h)=c\|h\|$ respectively. Then we obtain 5.2) from (5.1).

REMARK 5.1. The interpolating operator $T_{h}$ considered in [12, Chapter 3] satisfies condition 2) of Lemma 5.1 .

Two models of functional dependence in partial differential equations are used in the literature. There are papers concerning the nonlinear equation

$$
\partial_{t} z(t, x)=G\left(t, x,(V z)(t, x), \partial_{x} z(t, x)\right)
$$

with the initial conditions

$$
z(t, x)=\varphi(t, x) \quad \text { on } E_{0}
$$

where $V$ is a Volterra type operator and $\varphi: E_{0} \rightarrow \mathbb{R}, G: E \times \mathbb{R} \times \mathbb{R}^{n} \rightarrow \mathbb{R}$. The main assumptions in existence theorems for (5.3), (5.4) concern the operator $V$. They are formulated in terms of inequalities for norms in some function spaces. A new model of functional dependence for initial value problems with solutions defined on the Haar pyramid is proposed in [21, [22]. Our results on implicit difference methods are based on that idea.

The paper [13] concerns implicit difference methods for (5.3), (5.4). The following property of the operator $V z$ is important in [13]: the interpolating operator $V_{h} z$ corresponding to $V$ satisfies the Lipschitz condition with respect to $z$. Let us consider problem (5.3), (5.4) with

$$
(V z)(t, x)=z(t-\tau, y) \cdot z(t-\tilde{\tau}, y)
$$


where $\tau, \tilde{\tau} \in\left[0, b_{0}\right]$. The results of [13] are not applicable to $(5.3),(5.4)$ with $V$ defined by (5.5). The interpolating operators $V_{h} z$ corresponding to (5.5) do not satisfy the Lipschitz condition. Note that Theorem 4.1 can be applied to the above Cauchy problem.

Let us consider problem (5.3), (5.4) with

$$
(V z)(t, x)=\int_{D[t, x]} z^{2}(\tau, y) d y d \tau
$$

The interpolating operators $V_{h} z$ corresponding to 5.6 do not satisfy the Lipschitz condition. Thus the results of [13] are not applicable to (5.3), (5.4) with $V$ given by (5.6). Note that Theorem 4.1 can be applied to the above Cauchy problem.

Now we present numerical examples. We apply the results of Section 4 to a differential equation with deviated variables and to a differential integral problem. For $n=2$ we put $E_{0}=\{0\} \times[-b, b] \times[-b, b], M>0$ and

$$
E=\left\{(t, x, y) \in \mathbb{R}^{3}: 0 \leq t<a, x, y \in[-b+M t, b-M t]\right\} .
$$

Initial value problems considered here have solutions on $E$. Let us write $h=$ $\left(h_{0}, h_{1}, h_{2}\right)$ and assume $h_{1}=h_{2}$. Let $N_{r} \in \mathbb{N}$ be defined by $x^{\left(m_{1}\right)}, y^{\left(m_{2}\right)} \in I[r]$ for $m_{1}, m_{2}=-N_{r},-N_{r}+1, \ldots, N_{r}-1, N_{r}$, where $I[r]=\left[-1+2 t^{(r)}, 1-2 t^{(r)}\right]$ and $x^{\left(N_{r}+1\right)}, y^{\left(N_{r}+1\right)} \notin I[r]$.

EXAMPLE 5.1. Consider the differential equation with deviated variables

$$
\begin{aligned}
= & \cos \left(x \partial_{x} z(t, x, y)-y \partial_{y} z(t, x, y)\right)-x \partial_{x} z(t, x, y)-y \partial_{y} z(t, x, y) \\
& +z(t, 0.5(x+y), 0.5(x-y))+x y(1+2 t) z(t, x, y)-\exp \left(0.25 t\left(x^{2}-y^{2}\right)\right)
\end{aligned}
$$

with the initial condition

$$
z(0, x, y)=1 \quad \text { on } E_{0} .
$$

We put $a=0.4, b=1, M=2$ in (5.7). The solution of (5.8), (5.9) is known, it is $v(t, x, y)=e^{t x y}$. Let us denote by $z_{h}: E_{h} \rightarrow \mathbb{R}$ the solution of the implicit difference problem corresponding to (5.8), (5.9). Write

$$
\epsilon_{h}^{(r)}=\frac{1}{\left(2 N_{r}+1\right)^{2}} \sum_{m_{1}=-N_{r}}^{N_{r}} \sum_{m_{2}=-N_{r}}^{N_{r}}\left|v_{h}^{(r, m)}-z_{h}^{(r, m)}\right| .
$$

The number $\epsilon_{h}^{(r)}$ is the arithmetical mean of the error with fixed $t^{(r)}$. We give experimental values of the above defined errors for $h_{0}=0.01, h_{1}=h_{2}=$ 0.001 .

\begin{tabular}{ccccccc}
\hline$t^{(r)}$ & 0.15 & 0.20 & 0.25 & 0.30 & 0.35 & 0.40 \\
$\epsilon_{h}^{(r)}$ & 0.000785 & 0.000941 & 0.000975 & 0.000929 & 0.000918 & 0.001150 \\
\hline
\end{tabular}


The results in the table are consistent with our theoretical analysis. We have solved problem (5.8), (5.9) by using an explicit Lax scheme with the same steps of the mesh. In the case in question the (CFL) condition is not satisfied and the errors are greater than 100 for $r \geq 10$.

EXAmple 5.2. Consider the differential integral equation

$$
\begin{aligned}
= & -0.25 x \partial_{x} z(t, x, y)+0.25 x \cos \left(\partial_{x} z(t, x, y)+\int_{0}^{x} z(t, s, y) d s\right) \\
& -0.25 y \partial_{y} z(t, x, y)-0.25 y \sin \left(\partial_{y} z(t, x, y)+\int_{0}^{y} z(t, x, s) d s\right)+g(t, x, y)
\end{aligned}
$$

where $g(t, x, y)=\cos t \cos x \cos y-x \sin t \sin x \cos y-y \sin t \cos x \sin y-0.25 x$, with the initial condition

$$
z(0, x, y)=0 \quad \text { on } E_{0} .
$$

We put $a=1.5, b=1, M=0.5$ in (5.7). The solution to this problem is $v(t, x, y)=\sin t \cos x \cos y$. Let us denote by $z_{h}: E_{h} \rightarrow \mathbb{R}$ the solution of the implicit difference problem corresponding to 5.11), 5.12). Let $\epsilon_{h}^{(r)}$ be defined by $(5.10)$. In the table we give experimental values of the above defined errors for $h_{0}=0.02, h_{1}=h_{2}=0.002$.

\begin{tabular}{ccccccc}
\hline$t^{(r)}$ & 0.54 & 0.60 & 0.64 & 0.70 & 0.74 & 0.80 \\
$\epsilon_{h}^{(r)}$ & 0.006636 & 0.007498 & 0.008044 & 0.008811 & 0.009283 & 0.009927 \\
\hline
\end{tabular}

The results in the table are consistent with our theoretical analysis. We have solved problem (5.8), (5.9) by using an explicit Lax scheme with the same steps of the mesh. In this case the (CFL) condition is not satisfied and the errors are greater than 100 for $r \geq 40$.

Note that we have a little better results for the differential equation with deviated variables than for the differential integral problem. This is due to the fact that we use interpolating values $V_{h} z_{h}$ at the points $0.5\left(x^{\left(m_{1}\right)}+y^{\left(m_{2}\right)}\right)$, $0.5\left(x^{\left(m_{1}\right)}-y^{\left(m_{2}\right)}\right)$ in the first example and we use interpolating values $V_{h} z_{h}$ on the intervals $\left[0, x^{\left(m_{1}\right)}\right],\left[0, y^{\left(m_{2}\right)}\right]$ in the second example.

The above examples show that there are implicit difference methods for functional differential equations which are convergent and the corresponding explicit difference schemes are not convergent. Difference schemes obtained by the discretization (1.1), (1.2) have the following property: a large number of previous values $z_{h}^{(r, m)}, u_{h}^{(r, m)}$ must be preserved because they are needed to compute an approximate solution $\left(z_{h}, u_{h}\right)$ for $t=t^{(r+1)}$. 


\section{References}

[1] P. Bassanini and M. C. Salvadori, Un problema ai limiti per sistemi integrodifferenziali nonlineari di tipo iperbolico, Boll. Un. Mat. Ital. B 5 (1981), 785-798.

[2] P. Brandi and R. Ceppitelli, Existence, uniqueness and continuous dependence for a hereditary nonlinear functional partial differential equation of the first order, Ann. Polon. Math. 47 (1986), 121-136.

[3] P. Brandi and C. Marcelli, Haar inequality in hereditary setting and applications, Rend. Sem. Mat. Univ. Padova 96 (1996), 177-194.

[4] W. Czernous and Z. Kamont, Implicit difference methods for Hamilton-Jacobi functional differential equations, Sib. Zh. Vychisl. Mat. 12 (2009), no. 1, 57-70.

[5] T. Człapiński, On the local Cauchy problem for nonlinear hyperbolic functionaldifferential equations, Ann. Polon. Math. 67 (1997), 215-232.

[6] W. Eichhorn and W. Gleissner, On a functional differential equation arising in the theory of the distribution of wealth, Aequationes Math. 28 (1985), 190-198.

[7] M. El Doma, Analysis of nonlinear integro-differential equations arising in the agedependent epidemic models, Nonlinear Anal. 11 (1987), 913-937.

[8] E. Godlewski and P. Raviart, Numerical Approximation of Hyperbolic Systems of Conservation Laws, Springer, Berlin, 1996.

[9] D. Gottlieb and E. Tadmor, The CFL condition for spectral approximations to hyperbolic initial-boundary value problems, Math. Comput. 56 (1991), 565-588.

[10] D. Greenhalgh and I. Gyôri, Existence and uniqueness of solutions of a perturbation of the McKendrick-Von Foester equation, Functional Differential Equations 11 (2004), 69-76.

[11] D. Jaruszewska-Walczak and Z. Kamont, Numerical methods for hyperbolic functional differential problems on the Haar pyramid, Computing 65 (2000), 45-72.

[12] Z. Kamont, Hyperbolic Functional Differential Inequalities and Applications, Kluwer, Dordrecht, 1999.

[13] A. Kępczyńska, Implicit difference methods for Hamilton-Jacobi differential functional equations, Demonstratio Math. 40 (2007), 125-150.

[14] K. Kropielnicka, Implicit difference methods for quasilinear parabolic functional differential problems of the Dirichlet type, Appl. Math. (Warsaw) 35 (2008), 155-175.

[15] K. M. Magomedov and A. S. Kholodov, Difference-Characteristic Numerical Methods, Nauka, Moscow, 1988 (in Russian).

[16] M. Malec, Sur une famille bi-paramétrique des schémas des différences finies pour les systèmes paraboliques, Bull. Acad. Polon. Sci. Sér. Sci. Math. Astronom. Phys. 23 (1975), 871-875.

[17] —, Sur une famille biparamétrique de schémas des différences finies pour un système d'équations paraboliques aux dérivées mixtes et avec des conditions aux limites du type de Neumann, Ann. Polon. Math. 32 (1976), 33-42.

[18] C. V. Pao, Numerical methods for systems of nonlinear parabolic equations with time delays, J. Math. Anal. Appl. 240 (1999), 249-279.

[19] - Finite difference solutions of reaction diffusion equations with continuous time delays, Comput. Math. Appl. 42 (2001), 399-412.

[20] K. Prządka, The finite difference method in partial differential-functional equations, Univ. Iagel. Acta Math. 29 (1992), 41-60.

[21] E. Puźniakowska, Classical solutions of quasilinear functional differential systems on the Haar pyramid, Differential Equations Appl. 1 (2009), 179-197.

[22] E. Puźniakowska-Gałuch, On the local Cauchy problem for first order partial differential functional equations, Ann. Polon. Math. 98 (2010), 39-61. 
[23] A. A. Samarskii, The Theory of Difference Schemes, Dekker, New York, 2001.

[24] A. A. Samarskii, P. P. Matus and P. N. Vabishchevich, Difference Schemes with Operator Factor, Kluwer, Dordrecht, 2002.

[25] E. Sinestrari and G. F. Webb, Nonlinear hyperbolic systems with nonlocal boundary conditions, J. Math. Anal. Appl. 121 (1987), 449-464.

[26] J. Szarski, On integro-differential equations, Ann. Polon. Math. 14 (1964), 321-333.

[27] Y.-M. Wang and C. V. Pao, Time - delay finite difference reaction-diffusion systems with nonquasimonotone functions, Numer. Math. 103 (2006), 485-513.

[28] J. Wu, Theory and Applications of Partial Functional-Differential Equations, Springer, New York, 1996.

Elżbieta Puźniakowska-Gałuch

Institute of Mathematics

University of Gdańsk

Wit Stwosz St. 57

80-952 Gdańsk, Poland

E-mail: Elzbieta.Puzniakowska@mat.ug.edu.pl

Received on 11.12.2009;

revised version on 28.5.2010 